\title{
Permanence of Structured Population Models Governed by ODEs and the Basic Reproduction Number
}

\author{
Ryusuke KoN \\ Department of Biology, Kyushu University \\ Hakozaki 6-10-1, Higashi-ku, Fukuoka 812-8581, Japan \\ E-mail:kon-r@math.club.ne.jp
}

Received May 26, 2006

Revised September 22, 2006

\begin{abstract}
This paper considers the dynamics of a general nonlinear structured population model governed by ordinary differential equations. We are especially concerned with the survival possibility of structured populations. Our results show that, under a certain mild condition, the instability of the population free equilibrium point implies that the structured population survives in the sense of permanence. Furthermore, the relationship between the basic reproduction number and the instability of the population free equilibrium point provides simple criteria for population survival. The results are applied to both stage-structured and spatially structured models.
\end{abstract}

Key words: average Liapunov functions, exponentially nonnegative matrices, ordinary differential equations

\section{Introduction}

One of the important properties of a population model is the stability of the population free (or extinction) equilibrium. When the population free equilibrium is unstable, we expect that the population survives. If a population model is simple, this expectation is easily validated. For example, consider the logistic equation

$$
\dot{x}=r\left(1-\frac{x}{K}\right) x
$$

where the variable $x$ denotes the total population density, and the parameters $r$ and $K$ denote the intrinsic growth rate and the carrying capacity of the population, respectively. It is clear that the population survives if $r>0$ and $K>0$. In fact, if $r>0$ and $K>0$, then the population free equilibrium point $x=0$ is unstable and this instability leads to the globally asymptotically stable positive equilibrium point $x=K$. On the other hand, if a population model is complex, it is not clear whether the instability of the population free equilibrium implies that the population survives. For example, consider a high dimensional system of the population dynamics. Then the population free equilibrium could be saddle. Therefore, even if it is unstable, it could attract some orbits starting at the state where the population density is positive. The purpose of this paper is to provide a condition under which the survival possibility of a population is completely determined by the instability of the population free equilibrium.

One way to generalize the logistic equation is to incorporate the heterogeneity of individuals within a population. In the logistic equation, all individuals within 
the population are treated as identical. However, individuals in biological populations are not homogeneous. They are distinct with regard to their physiological and behavioral characteristics. Such distinctions are influential to the vital processes such as birth, death and growth. Especially, the heterogeneity is crucially important when we consider the spread of infectious diseases since the population is divided into at least two classes: the classes of susceptibles and infectives.

The population model taking into account the heterogeneity of individuals within a population is called the structured population model. There are several frameworks for structured population models (e.g., see $[3,4,6,14]$ ). One of such frameworks has the following form

$$
\dot{\mathbf{x}}=A_{\mathbf{x}} \mathbf{x}
$$

where $\mathbf{x}=\left(x_{1}, x_{2}, \ldots, x_{n}\right)^{\top}, \dot{\mathbf{x}}=\left(d x_{1} / d t, d x_{2} / d t, \ldots, d x_{n} / d t\right)^{\top}$ and $A_{\mathbf{x}}=\left(a_{i j}(\mathbf{x})\right)$ is an $n \times n$ matrix. In this equation, the population is divided into $n$ classes according to some discretized property of heterogeneous individuals (e.g., developmental stage, spatial location or infection stage). Each variable $x_{i}$ represents the population density of class $i$. We can find many specific examples of (1) in the literature (e.g., see $[13,17])$.

This paper considers the survival possibility of the structured population in system (1). Since each variable $x_{i}$ represents a population density, we are interested only in the solutions of (1) in the nonnegative cone $\mathbb{R}_{+}^{n}=\left\{\mathbf{x} \in \mathbb{R}^{n}: x_{1} \geq 0, x_{2} \geq\right.$ $\left.0, \ldots, x_{n} \geq 0\right\}$. Furthermore, we are not concerned with a system with unbounded solutions. We concentrate on system (1) with the dissipativity, which is defined as follows.

Definition 1 (dissipativity). System (1) is said to be dissipative if there exists a compact set $X \subset \mathbb{R}_{+}^{n}$ such that for every $\mathbf{x}(0) \in \mathbb{R}_{+}^{n}$ there exists a $T=$ $T(\mathbf{x}(0)) \geq 0$ satisfying $\mathbf{x}(t) \in X$ for all $t \geq T$.

One way to evaluate the possibility of population survival is to investigate the dynamical property defined as follows.

Definition 2 (p-permanence). Let $N=\{1,2, \ldots, n\}$ and $J \subset N$. System (1) is said to be p-permanent with respect to $J$ if it is dissipative and there exists a positive constant $\delta>0$ such that

$$
\liminf _{t \rightarrow \infty} \sum_{i \in J} x_{i}(t) \geq \delta
$$

holds for all $\mathbf{x}(0) \in \mathbb{R}_{+}^{n} \backslash O_{J}$, where $O_{J}=\left\{\mathbf{x} \in \mathbb{R}_{+}^{n}: x_{i}=0\right.$ for all $\left.i \in J\right\}$.

By this definition, p-permanence with respect to $J$ ensures that the sum of the population densities $x_{i}, i \in J$, is bounded below from zero. Thus, a part of the structured population associated with $J$ survives as long as it presents initially, i.e., $\sum_{i \in J} x_{i}(0)>0$ (if $J=N$, then the total population survives). Note that each 
class corresponding to $J$ does not necessarily survive even if (1) is p-permanent with respect to $J$ because some $x_{i}, j \in J$, could approach zero.

We introduce another definition associated with the population survival as follows.

Definition 3 (c-permanence). Let $N=\{1,2, \ldots, n\}$ and $J \subset N$. System (1) is said to be c-permanent with respect to $J$ if it is dissipative and there exists a positive constant $\delta>0$ such that

$$
\liminf _{t \rightarrow \infty} x_{i}(t) \geq \delta, \quad i \in J
$$

holds for all $\mathbf{x}(0) \in \mathbb{R}_{+}^{n} \backslash O_{J}$, where $O_{J}=\left\{\mathbf{x} \in \mathbb{R}_{+}^{n}: x_{i}=0\right.$ for all $\left.i \in J\right\}$.

If system (1) is c-permanent with respect to $J$, then it is ensured that each population density $x_{i}, i \in J$, does not approach zero (note that each $x_{i}$ is bounded above). That is, coexistence of all classes is guaranteed. If $J$ is identical to $N$, then we omit the part "with respect to $N$." In this paper, we focus on these dynamical properties ( $\mathrm{p}$ - and c-permanence) and provide a condition under which the instability of the population free equilibrium point $\mathbf{x}=0$ implies $\mathrm{p}$ - or c-permanence of (1).

This paper is organized as follows. In Section 2, we introduce some useful notation used throughout in this paper. Since system (1) includes biologically unrealistic cases, we introduce some assumptions for (1) in Section 3. Section 4 includes the main results of this paper. The main results show that, under some mild condition, system (1) is c-permanent if the population free equilibrium point $\mathbf{x}=0$ is unstable. This result is analogous to the result for the logistic equation. In Section 5 , in order to evaluate the instability of the population free equilibrium point $\mathbf{x}=0$, we introduce the basic reproduction number $\mathcal{R}_{0}$. We show the mathematical relationship between $\mathcal{R}_{0}$ and the instability of $\mathbf{x}=0$. In Section 6 , to illustrate our results, we apply them to two specific models: stage-structured and spatially structured population models. The final section includes concluding remarks. Some mathematical theorems are listed in Appendices.

\section{Preliminaries}

Terminology concerning dynamical systems is given in Appendix A.

Both the zero vector and the zero matrix are denoted by 0 . For vectors $\mathbf{x}=$ $\left(x_{1}, x_{2}, \ldots, x_{n}\right)^{\top}$ and $\mathbf{y}=\left(y_{1}, y_{2} \ldots, y_{n}\right)^{\top}$, we write $\mathbf{x} \geq \mathbf{y}(\operatorname{resp} . \mathbf{x}>\mathbf{y})$ if $x_{i} \geq$ $y_{i}$ (resp. $x_{i}>y_{i}$ ) for all $i$. A vector $\mathbf{x}$ is called nonnegative (resp. positive) if $\mathbf{x} \geq 0$ (resp. $\mathbf{x}>0$ ). For matrices $A=\left(a_{i j}\right)$ and $B=\left(b_{i j}\right)$, we write $A \geq B$ (resp. $A>B$ ) if $a_{i j} \geq b_{i j}$ (resp. $a_{i j}>b_{i j}$ ) for all $i, j$. A matrix $A$ is called nonnegative (resp. positive) if $A \geq 0$ (resp. $A>0$ ). An $n \times n$ matrix $A=\left(a_{i j}\right)$ is said to be exponentially nonnegative if $a_{i j} \geq 0$ for all $i \neq j, 1 \leq i, j \leq n$, i.e., all off-diagonal entries are nonnegative. Appendix B includes some important properties of exponentially nonnegative matrices. The directed graph of an $n \times n$ matrix $A=\left(a_{i j}\right)$ is denoted by $G(A)$, which is obtained by drawing an arrow from 
$j$ to $i$ whenever $a_{i j} \neq 0$. For $i, j \in\{1,2 \ldots, n\}, j$ is said to be accessible to $i$ in $G(A)$ if there is a path from $j$ to $i$ in $G(A)$, i.e., $a_{i k_{1}} a_{k_{1} k_{2}} \cdots a_{k_{l} j} \neq 0$ for some $k_{1}, k_{2}, \ldots, k_{l} \in\{1,2, \ldots, n\}$.

Let bd $\mathbb{R}_{+}^{n}=\left\{\mathbf{x} \in \mathbb{R}_{+}^{n}: x_{1} x_{2} \cdots x_{n}=0\right\}$. Let $N=\{1,2, \ldots, n\}$ and $J \subset N$ with $J \neq \emptyset$. Let $O$ be the set consisting only of the origin and $O_{J}=\left\{\mathbf{x} \in \mathbb{R}_{+}^{n}: x_{i}=\right.$ 0 for all $i \in J\}$. Thus, $O_{J} \subset$ bd $\mathbb{R}_{+}^{n}$ and $O=O_{N}$. $A^{(J)}$ is defined to be the submatrix of $A$ composed of $a_{i j}, i, j \in J$. The identity matrix is denoted by $I$. For real numbers $a$ and $b$, we write $\operatorname{sign}(a)=\operatorname{sign}(b)$ if $a$ and $b$ have the same sign,- 0 or + .

The set of eigenvalues of a matrix $A$ is called the spectrum of $A$ and denoted by $\sigma(A)$. The stability modulus of a matrix $A$ is defined by

$$
\mu(A)=\max \{\operatorname{Re} \lambda: \lambda \in \sigma(A)\},
$$

where $\operatorname{Re} \lambda$ denotes the real part of $\lambda$. A matrix $A$ is said to be unstable (resp. stable) if $\mu(A)>0$ (resp. $\mu(A)<0$ ). The spectral radius of a matrix $A$ is defined by

$$
\rho(A)=\max \{|\lambda|: \lambda \in \sigma(A)\} .
$$

\section{Assumptions}

We assume that system (1) satisfies the following conditions:

(H1) Each $a_{i j}: \mathbb{R}_{+}^{n} \rightarrow \mathbb{R}$ is continuously differentiable.

(H2) Each off-diagonal entry of $A_{\mathbf{x}}$ does not change its sign $\left(-, 0\right.$ or + ) on $\mathbb{R}_{+}^{n}$, i.e., $\operatorname{sign}\left(a_{i j}(\mathbf{x})\right)=\operatorname{sign}\left(a_{i j}(0)\right), i \neq j$, for all $\mathbf{x} \in \mathbb{R}_{+}^{n}$.

(H3) $\quad A_{0}=\left(a_{i j}(0)\right)$ is exponentially nonnegative.

(H4) System (1) is dissipative.

(H1) ensures that there exists a unique solution of (1), which is locally defined. (H2) means that each interaction between classes does not change qualitatively. Under the assumptions (H1) and (H2), the condition (H3) is necessary and sufficient for forward invariance of $\mathbb{R}_{+}^{n}$.

Proposition 4. Assume that (H1) and (H2) hold. Then the nonnegative cone $\mathbb{R}_{+}^{n}$ is forward invariant if and only if (H3) holds. The assumption (H3) also ensures that if $x_{k}(0)>0$, then $x_{k}(t)>0$ for all $t>0$.

Proof. Suppose that (H3) holds. Let $\mathbf{x} \in \mathrm{bd} \mathbb{R}_{+}^{n}$ with $x_{k}=0$. Since $A_{\mathbf{x}}$ is exponentially nonnegative, every off-diagonal entry $a_{i j}(\mathbf{x})$ of $A_{\mathbf{x}}$ is nonnegative. Therefore, we have

$$
\dot{x}_{k}=\sum_{j=1}^{n} a_{k j}(\mathbf{x}) x_{j} \geq 0 .
$$

Note that $a_{k k}(\mathbf{x}) x_{k}=0$. It follows from Theorem 18 of Appendix $\mathrm{A}$ that $\mathbb{R}_{+}^{n}$ is forward invariant.

Suppose that (H3) does not hold. Then there exist $k, l \in N, k \neq l$ such that $a_{k l}(0)<0$. We choose $\mathbf{x} \in \operatorname{bd} \mathbb{R}_{+}^{n}$ satisfying $x_{l}>0$ and $x_{i}=0$ for all $i \neq l$. Then, by (H2), $\dot{x}_{k}=a_{k l}(\mathbf{x}) x_{l}<0$ holds. This implies that $\mathbb{R}_{+}^{n}$ is not forward invariant. 
Finally, consider the last statement. Let $\mathbf{x}(t)$ be a solution of (1) with $\mathbf{x}(0) \in$ $\mathbb{R}_{+}^{n}$ and $x_{k}(0)>0$. Suppose that $x_{k}\left(t_{1}\right)=0$ for some $t_{1}>0$. Divide $N$ into two groups, $J_{0}=\left\{i \in N: x_{i}\left(t_{1}\right)=0\right\}$ and $J_{+}=\left\{i \in N: x_{i}\left(t_{1}\right)>0\right\}$. If $\dot{x}_{i}\left(t_{1}\right)=0$ for all $i \in J_{0}$, then system (1) has a solution $\widetilde{\mathbf{x}}(t)$ which passes through the point $\mathbf{x}\left(t_{1}\right)$ and satisfies $\widetilde{x}_{i}(t) \equiv 0$ for all $i \in J_{0}$ since $a_{i j}(\mathbf{x})=0, i \in J_{0}, j \in J_{+}$, for all $\mathbf{x} \in \mathbb{R}_{+}^{n}$. This is a contradiction to the uniqueness of the solutions (note that $\left.k \in J_{0}\right)$. If $\dot{x}_{i}\left(t_{1}\right) \neq 0$ for some $i \in J_{0}$, then $x_{i}(t)<0$ for some $t \in\left(0, t_{1}\right)$. This is a contradiction to the forward invariance of $\mathbb{R}_{+}^{n}$.

The following proposition provides a sufficient condition for (H4).

Proposition 5. Assume that (H1)-(H3) hold. If there exist constants $K>0$ and $\lambda_{\infty}<0$ such that the inequalities $\sum_{i=1}^{n} a_{i j}(\mathbf{x}) \leq \lambda_{\infty}, j=1,2, \ldots, n$, hold for all $\mathbf{x} \in \mathbb{R}_{+}^{n}$ with $\sum_{i=1}^{n} x_{i} \geq K$, then system (1) is dissipative.

Proof. Let $\mathbf{x}(t)$ be a solution of (1) with $\mathbf{x}(0) \in \mathbb{R}_{+}^{n}$. Suppose that $\sum_{i=1}^{n} x_{i}(t) \geq K$ for all $t \geq 0$. Then the solution satisfies

$$
\begin{aligned}
\sum_{i=1}^{n} \dot{x}_{i}(t) & =\sum_{i=1}^{n} \sum_{j=1}^{n} a_{i j}(\mathbf{x}(t)) x_{j}(t) \\
& =\sum_{i=1}^{n} a_{i 1}(\mathbf{x}(t)) x_{1}(t)+\sum_{i=1}^{n} a_{i 2}(\mathbf{x}(t)) x_{2}(t)+\cdots+\sum_{i=1}^{n} a_{i n}(\mathbf{x}(t)) x_{n}(t) \\
& \leq \lambda_{\infty} \sum_{i=1}^{n} x_{i}(t)
\end{aligned}
$$

It follows from $\lambda_{\infty}<0$ that $\mathbf{x}(t) \rightarrow 0$ as $t \rightarrow \infty$. This is a contradiction. Hence, for every $\mathbf{x}(0) \in \mathbb{R}_{+}^{n}$ there exists a $T \geq 0$ such that $\sum_{i=1}^{n} x_{i}(T)<K$.

Let $\mathbf{x}(0) \in \mathbb{R}_{+}^{n}$ with $\sum_{i=1}^{n} x_{i}(0) \leq K$. Suppose that $\sum_{i=1}^{n} x_{i}\left(T^{\prime}\right)=K+\epsilon$ for some $T^{\prime}>0$ and $\epsilon>0$. Then we can choose $t_{0}, t_{1} \in\left[0, T^{\prime}\right], t_{0}<t_{1}$, such that $\sum_{i=1}^{n} x_{i}\left(t_{0}\right)=K, \sum_{i=1}^{n} x_{i}\left(t_{1}\right)=K+\epsilon$ and $K<\sum_{i=1}^{n} x_{i}(t)<K+\epsilon$ for all $t \in\left(t_{0}, t_{1}\right)$. By the mean value theorem, there exists a $T^{\prime \prime} \in\left(t_{0}, t_{1}\right)$ such that

$$
\sum_{i=1}^{n} \dot{x}_{i}\left(T^{\prime \prime}\right)=\frac{\sum_{i=1}^{n} x_{i}\left(t_{1}\right)-\sum_{i=1}^{n} x_{i}\left(t_{0}\right)}{t_{1}-t_{0}}=\frac{\epsilon}{t_{1}-t_{0}}>0 .
$$

This is a contradiction since $\sum_{i=1}^{n} x_{i}\left(T^{\prime \prime}\right)>K$ implies $\sum_{i=1}^{n} \dot{x}_{i}\left(T^{\prime \prime}\right)<0$. Therefore, the compact set $\left\{\mathrm{x} \in \mathbb{R}_{+}^{n}: \sum_{i=1}^{n} x_{i} \leq K\right\}$ is a forward invariant set attracting every point $\mathrm{x} \in \mathbb{R}_{+}^{n}$.

Note that, under the assumptions (H1)-(H4), it is ensured that, for every $\mathbf{x}(0) \in \mathbb{R}_{+}^{n}$, there exists a unique solution $\mathbf{x}(t)$ of $(1)$, which is defined for all $t \geq 0$.

\section{Dynamics of an irreducible subsystem}

The directed graph $G\left(A_{0}\right)$ reflects some structure of (1). According to the graph $G\left(A_{0}\right)$, we can divide the structured model into some irreducible subsystems. 
In this section, we consider permanence of such an irreducible subsystem. The next section consider the dynamics of the full system.

Before the main results (Theorems 8 and 10), we will obtain two lemmas for some properties of system (1).

Lemma 6. Assume that (H1)-(H4) hold. Let $J \subset N$. If $J=N$, then $O_{J}=O$ is invariant. If $J \neq N, \emptyset$ and $a_{i j}(0)=0$ for all $i \in J$ and $j \in N \backslash J$, then $O_{J}$ and $\mathbb{R}_{+}^{n} \backslash O_{J}$ are forward invariant.

Proof. The first statement obviously holds since the origin is an equilibrium point of (1). Suppose that $J \neq N, \emptyset$ and $a_{i j}(0)=0$ for all $i \in J$ and $j \in N \backslash J$. Then, by $(\mathrm{H} 2), a_{i j}(\mathbf{x})=0, i \in J, j \in N \backslash J$, also holds for all $\mathbf{x} \in \mathbb{R}_{+}^{n}$. Hence, for every $\mathbf{x} \in O_{J}$

$$
\dot{x}_{i}=\sum_{j \in N \backslash J} a_{i j}(\mathbf{x}) x_{j}=0, \quad i \in J .
$$

This implies that $O_{J}$ and $\mathbb{R}_{+}^{n} \backslash O_{J}$ are forward invariant. Otherwise, the initial value problem does not have a unique solution.

Lemma 7. Assume that $(\mathrm{H} 1)-(\mathrm{H} 4)$ hold. Let $J \subset N$ and $J \neq \emptyset$. Suppose that $A_{0}^{(J)}$ is irreducible. Then $x_{i}(t)>0, i \in J$, for all $t>0$ provided $\mathbf{x}(0) \in \mathbb{R}_{+}^{n} \backslash O_{J}$.

Proof. Suppose $J \neq N$. Without loss of generality, we can assume that $J=\{1,2, \ldots, k\}, 1 \leq k \leq n$. Let $\mathbf{x}(t)$ be a solution of (1) with $\mathbf{x}(0) \in \mathbb{R}_{+}^{n} \backslash O_{J}$ and $\mathbf{x}^{(J)}(t)=\left(x_{1}(t), x_{2}(t), \ldots, x_{k}(t)\right)^{\top}$. Then $\mathbf{x}(t)$ is nonnegative for all $t \geq 0$ and satisfies

$$
\dot{\mathbf{x}}^{(J)}(t)=A_{\mathbf{x}(t)}^{(J)} \mathbf{x}^{(J)}(t)+\mathbf{b}(t)
$$

where $\mathbf{b}(t)=\left(b_{1}(t), b_{2}(t), \ldots, b_{k}(t)\right)^{\top}$ and $b_{i}(t)=\sum_{j \in N \backslash J} a_{i j}(\mathbf{x}(t)) x_{j}(t)$. Since $A_{\mathbf{x}(t)}$ is exponentially nonnegative, $\mathbf{b}(t) \geq 0$ for all $t \geq 0$. By assumption, we can choose an exponentially nonnegative irreducible matrix $B$ satisfying $A_{\mathbf{x}(t)}^{(J)} \geq B$ for all $t \in[0,1]$. Let $\mathbf{c}(t)=\left(A_{\mathbf{x}(t)}^{(J)}-B\right) \mathbf{x}^{(J)}(t)+\mathbf{b}(t)$. Then $\mathbf{x}^{(J)}(t)$ satisfies

$$
\mathbf{x}^{(J)}(t)=e^{t B} \mathbf{x}^{(J)}(0)+\int_{0}^{t} e^{(t-s) B} \mathbf{c}(s) d s, \quad t \in[0,1] .
$$

Since $B$ is exponentially nonnegative and irreducible, $e^{t B}>0$ for all $t>0$ (e.g., see Theorem 8.2 of [19]). Therefore, (2) with Proposition 4 implies that $x_{i}(t)>0$, $i \in J$, for all $t>0$.

The following theorem is our main result and provides a condition for p-permanence of subsystems of (1). Conditions (i) and (ii) are valid for (1) with stage-structure and spatial structure, respectively (see Section 7).

Theorem 8. Assume that (H1)-(H4) hold, and that one of the following two conditions is satisfied: 
(i) $\omega\left(O_{J}\right)=O$ or

(ii) $A_{\mathbf{x}}^{(J)}=A_{0}^{(J)}$ for all $\mathbf{x} \in O_{J}$,

where $\omega\left(O_{J}\right)$ is the $\omega$-limit set of $O_{J}$ (see Appendix A) and $J \subset N$ with $J \neq \emptyset$. Suppose that either $J=N$ or $a_{i j}(0)=0$ for all $i \in J$ and $j \in N \backslash J$, and that $A_{0}^{(J)}$ has a positive eigenvalue and corresponding positive eigenvector (i.e., it is sufficient that $A_{0}^{(J)}$ is irreducible; see Theorem 21 of Appendix B). Then system (1) is p-permanent with respect to $J$ if $\mu\left(A_{0}^{(J)}\right)>0$.

Proof. Without loss of generality, we can assume that $J=\{1,2, \ldots, k\}, 1 \leq$ $k \leq n$. Since system (1) is dissipative, Theorem 19 of Appendix A guarantees that there exists a compact absorbing set $X \subset \mathbb{R}_{+}^{n}$ for $\mathbb{R}_{+}^{n}$, i.e., all orbits in $\mathbb{R}_{+}^{n}$ ultimately enter the compact forward invariant set $X$. Therefore, it is enough to focus on the orbits in $X$. By Lemma 6, both $O_{J}$ and $X \backslash O_{J}$ are forward invariant. By constructing an average Liapunov function, we shall show that (1) is p-permanent with respect to $J$.

By assumption, there exists a positive vector $\mathbf{v}>0$ such that $\left(A_{0}^{(J)}\right)^{\top} \mathbf{v}=\lambda \mathbf{v}$, where $\lambda$ is a positive eigenvalue of $A_{0}^{(J)}$. Let $P(\mathbf{x})=\mathbf{v} \cdot \mathbf{x}^{(J)}$, where ". " denotes the inner product and $\mathbf{x}^{(J)}=\left(x_{1}, x_{2}, \ldots, x_{k}\right)^{\top}$. Then it is clear that $P: X \rightarrow \mathbb{R}_{+}$ is continuously differentiable. Furthermore, $P(\mathbf{x})=0$ if and only if $\mathbf{x} \in O_{J}$, i.e., condition (a) in Theorem 20 of Appendix A holds. Define $\psi: X \rightarrow \mathbb{R}$ by

$$
\psi(\mathbf{x})=\min _{i \in J} \frac{\left(\left(A_{\mathbf{x}}^{(J)}\right)^{\top} \mathbf{v}\right)_{i}}{v_{i}},
$$

where $\left(\left(A_{\mathbf{x}}^{(J)}\right)^{\top} \mathbf{v}\right)_{i}$ is the $i$-th component of the vector $\left(A_{\mathbf{x}}^{(J)}\right)^{\top} \mathbf{v}$. Then $\psi$ is continuous and

$$
\begin{aligned}
\dot{P}(\mathbf{x}) & \geq \mathbf{v} \cdot A_{\mathbf{x}}^{(J)} \mathbf{x}^{(J)} \\
& =\mathbf{x}^{(J)} \cdot\left(A_{\mathbf{x}}^{(J)}\right)^{\top} \mathbf{v} \\
& =\sum_{i \in J} v_{i} x_{i} \frac{\left(\left(A_{\mathbf{x}}^{(J)}\right)^{\top} \mathbf{v}\right)_{i}}{v_{i}} \geq P(\mathbf{x}) \psi(\mathbf{x})
\end{aligned}
$$

for all $\mathrm{x} \in X$.

If condition (i) holds, then it is clear that

$$
\sup _{t \geq 0} \int_{0}^{t} \psi(\mathbf{x}(s)) d s=\sup _{t \geq 0} \int_{0}^{t} \lambda d s>0
$$

holds for $\mathbf{x}(0) \in \omega\left(O_{J}\right)$. Furthermore, if condition (ii) holds, then (3) holds for all $\mathbf{x}(0) \in O_{J}$. This implies that system (1) is p-permanent with respect to $J$.

REMARK. An analogous result is found in [12], in which p-permanence of a discrete-time version of (1) is considered. By using Theorem 2 of [10], we can show 
that, under the assumption of Theorem 8, system (1) has an equilibrium point $\mathbf{x}^{*} \in \mathbb{R}_{+}^{n}$ with $x_{i}^{*}>0$ for some $i \in J$. Furthermore, $x_{i}^{*}>0$ for all $i \in J$ since $A_{0}^{(J)}$ is irreducible.

The following corollary is a special case of Theorem 8 .

Corollary 9. Assume that (H1)-(H4) hold. Suppose that $A_{0}$ is irreducible. Then system (1) is p-permanent if $\mu\left(A_{0}\right)>0$.

We see that p-permanence implies c-permanence in the following sense.

TheOrem 10. Assume that $(\mathrm{H} 1)-(\mathrm{H} 4)$ hold. Suppose that $A_{0}^{(J)}$ is irreducible for $J \subset N$ with $J \neq \emptyset$. Then system (1) is c-permanent with respect to $J$ if and only if it is p-permanent with respect to $J$.

Proof. Suppose that system (1) is p-permanent with respect to $J$. Then Theorem 19 of Appendix A shows that there exists a compact absorbing set $X$ for $\mathbb{R}_{+}^{n} \backslash O_{J}$ satisfying $X \cap O_{J}=\emptyset$. If $\phi_{t}(\mathbf{x})$ denotes the solution map for (1), then $X^{\prime}=\phi_{1}(X)$ is also a compact absorbing set for $\mathbb{R}_{+}^{n} \backslash O_{J}$. Moreover, Lemma 7 ensures that $\min _{i \in J} x_{i}>0$ holds for all $\mathrm{x} \in X^{\prime}$. Since $X^{\prime}$ is compact, there exists a $\delta>0$ such that $\min _{i \in J} x_{i} \geq \delta$ holds for every $\mathbf{x} \in X^{\prime}$. This implies that system (1) is c-permanent with respect to $J$.

It is clear that c-permanence with respect to $J$ implies p-permanence with respect to $J$.

\section{Dynamics of the full system}

If $A_{0}$ is irreducible, then c-permanence of (1) can be evaluated by Corollary 9 and Theorem 10. Furthermore, even if $A_{0}$ is reducible, c-permanence of some irreducible subsystem can be evaluated by Theorems 8 and 10. However, it is not clear whether the populations in the remaining subsystem of (1) can survive if some irreducible subsystem is c-permanent. In this section, we consider the destiny of a subsystem connecting to a surviving subsystem. The following theorem shows that the populations in the classes accessible from a surviving class also survive.

Theorem 11. Assume that (H1)-(H4) hold. Let $J \subset N, j \in J$ and $k \in$ $N \backslash J$. Suppose that $j$ is accessible to $k$ in $G\left(A_{0}\right)$. Then there exists a positive constant $\delta_{k}>0$ such that

$$
\liminf _{t \rightarrow \infty} x_{k}(t) \geq \delta_{k}
$$

holds for all $\mathbf{x}(0) \in \mathbb{R}_{+}^{n} \backslash O_{J}$ if there exists a positive constant $\delta_{j}>0$ such that $\liminf \operatorname{in}_{t \rightarrow \infty} x_{j}(t) \geq \delta_{j}$ holds for all $\mathbf{x}(0) \in \mathbb{R}_{+}^{n} \backslash O_{J}$.

Proof. Since $j$ is accessible to $k$ in $G\left(A_{0}\right), a_{k k_{1}}(0) a_{k_{1} k_{2}}(0) \cdots a_{k_{l} j}(0) \neq 0$ for some $k_{1}, k_{2}, \ldots, k_{l} \in N$. Without loss of generality, we can assume that $k, k_{1}, k_{2}, \ldots, k_{l}, j$ are pairwise distinct. By (H4) and the existence of $\delta_{j}>0$, Theorem 19 of Appendix A ensures that there exists a compact absorbing set $X_{j}$ for 
$\mathbb{R}_{+}^{n} \backslash O_{J}$ satisfying $X_{j} \cap O_{\{j\}}=\emptyset$. If $\phi_{t}(\mathbf{x})$ denotes the solution map for (1), then $X_{k_{l}}=\phi_{1}\left(X_{j}\right)$ is also a compact absorbing set for $\mathbb{R}_{+}^{n} \backslash O_{J}$. Let $\mathbf{x}(t)$ be a solution of system (1) with $\mathbf{x}(0) \in O_{\left\{k_{l}\right\}} \cap X_{j}$. Since $A_{\mathbf{x}(0)}$ is exponentially nonnegative and $a_{k_{l} j}(\mathbf{x}(0))>0$, we have

$$
\begin{aligned}
\dot{x}_{k_{l}}(0) & =a_{k_{l} 1}(\mathbf{x}(0)) x_{1}(0)+a_{k_{l} 2}(\mathbf{x}(0)) x_{2}(0)+\cdots+a_{k_{l} n}(\mathbf{x}(0)) x_{n}(0) \\
& \geq a_{k_{l} j}(\mathbf{x}(0)) x_{j}(0)>0
\end{aligned}
$$

Note that $x_{j}(0)>0$. The inequality $\dot{x}_{k_{l}}(0)>0$ implies that $X_{j} \backslash O_{\left\{k_{l}\right\}}$ is forward invariant and $\mathbf{x} \in O_{\left\{k_{l}\right\}} \cap X_{j}$ implies $\gamma^{+}(\mathbf{x}) \cap X_{j} \backslash O_{\left\{k_{l}\right\}} \neq \emptyset$. Therefore, $X_{k_{l}} \cap O_{\left\{k_{l}\right\}}=$ $\emptyset$. If we replace $j$ and $k_{l}$ in the above argument by $k_{l}$ and $k_{l-1}$, respectively, then we obtain a compact absorbing set $X_{k_{l-1}}$ for $\mathbb{R}_{+}^{n} \backslash O_{J}$ satisfying $X_{k_{l-1}} \cap O_{\left\{k_{l-1}\right\}}=\emptyset$. By repeating this argument, we eventually obtain the conclusion.

\section{The basic reproduction number}

In this section, we consider the basic reproduction number $\mathcal{R}_{0}$ of the following linear ordinary differential equation

$$
\dot{\mathbf{x}}=A_{0} \mathbf{x}
$$

This equation appears as the linearized system of (1) at the population free equilibrium point $\mathbf{x}=0$. The basic reproduction number $\mathcal{R}_{0}$ is usually defined to be the expected number of offspring per individual per life time. It is well known that this number plays an important role in the study of structured models. The basic reproduction number $\mathcal{R}_{0}$ can often be calculated and expressed in term of the entries in the matrix $A_{0}$ (see Chapter 5 of [6], and [7] for the definition of $\mathcal{R}_{0}$ for a more general class of structured models). Furthermore, the population free equilibrium point $\mathbf{x}=0$ is usually unstable (resp. stable) if $\mathcal{R}_{0}>1$ (resp. $\mathcal{R}_{0}<1$ ).

The mathematical foundation of such a relationship between the basic reproduction number and the instability of $\mathbf{x}=0$ has been established by Cushing and Yicang [5] (see also Section 1.1.2 of [4]). They established it for the framework of structured models described by difference equations. Furthermore, van den Driessche and Watmough [18] also proved the same relationship for ordinary differential equations with epidemiological models in mind. The purpose of this section is to introduce the similar result with system (4) in mind. Since our proof for this result is somewhat different from that in [18], the proof of our theorem is given in Appendix C.

We begin with the assumptions:

(A1) $\quad A_{0}$ is exponentially nonnegative irreducible $n \times n$ matrix.

(A2) $A_{0}=F_{0}+T_{0}$ holds for some nonnegative matrix $F_{0}$ and some exponentially nonnegative matrix $T_{0}$ satisfying $\mu\left(T_{0}\right)<0$.

(A3) $-F_{0} T_{0}^{-1}$ has a positive eigenvalue $\mathcal{R}_{0}$ such that $\mathcal{R}_{0}=\mu\left(-F_{0} T_{0}^{-1}\right)$ holds and to $\mathcal{R}_{0}$ there corresponds a nonnegative right eigenvector $\mathbf{w} \geq 0$. 
Note that $T_{0}^{-1}$ exists under the assumption (A2) (see Theorem 22 in Appendix B). Under these assumptions, we define the basic reproduction number as follows.

Definition 12. Assume that (A1)-(A3) hold and $-T_{0}^{-1} \mathbf{w}>0$. Then $\mathcal{R}_{0}$ is called the basic reproduction number of $A_{0}$.

The matrix $A_{0}$ of a structured model is usually divided into to two matrices: the matrix $F_{0}$ corresponding to the fertility and the remaining matrix $T_{0}=A_{0}-$ $F_{0}$. The matrix $T_{0}$ usually contains the terms corresponding to both death and transition between classes. The dynamics without the reproduction process is given by $\dot{\mathbf{x}}=T_{0} \mathbf{x}$. Therefore, the expected time that an individual starting in class $j$ will spend in class $i$ in the rest of its life is given by the $(i, j)$-entry of the $n \times n$ matrix

$$
\int_{0}^{\infty} e^{s T_{0}} d s=-T_{0}^{-1} .
$$

In fact, the probability that an individual starting in class $j$ exists in class $i$ after $s$ units of time is given by $e^{s T_{0}} \mathbf{y}$, where $\mathbf{y}$ is the unit vector whose $j$-th component equals 1 while all other components are zero (see Chapter 5 of [6]). If the fertility matrix $F_{0}$ has only one nonzero row in the first row, then $\mathcal{R}_{0}$ is equivalent to the $(1,1)$-entry of $-F_{0} T_{0}^{-1}$. This entry is surly equivalent to the expected number of offspring per newborn per life time if the first class corresponds to the newborn class. Therefore, in this case, the mathematical definition of $\mathcal{R}_{0}$ coincides with the biological meaning of the basic reproduction number (see Section 1.1.2 of [4] for the case where $F_{0}$ has multiple nonzero rows).

Concerning the relationship between $\mathcal{R}_{0}$ and the instability of $\mathbf{x}=0$, the following theorem holds (see Appendix C for its proof).

THEOREM 13. Assume that (A1)-(A3) hold and $-T_{0}^{-1} \mathbf{w}>0$. Then $\mu\left(A_{0}\right)>$ 0 (resp. $\mu\left(A_{0}\right)<0$ or $\mu\left(A_{0}\right)=0$ ) if and only if $\mathcal{R}_{0}>1$ (resp. $\mathcal{R}_{0}<1$ or $\mathcal{R}_{0}=1$ ).

It is worth noting that even if $\mathcal{R}_{0}$ does not have a correct biological meaning, due to the arbitrariness of $F_{0}$ and $T_{0}$, Theorem 13 provides a simple method evaluating the stability of the population free equilibrium point $\mathbf{x}=0$. Furthermore, an application of this theorem is not restricted to systems with the form (4) (see Appendix C).

\section{Applications}

In this section, we apply the results obtained in the previous sections to two specific structured models: a stage-structured model and a spatially structured model. 


\section{1. $\quad$ Stage-structured models}

Consider the following stage-structured model (the corresponding discrete-time model is called the Usher matrix model [4]):

$$
\left\{\begin{array}{c}
\dot{x}_{1}=\sum_{i=1}^{n} \beta_{i}(\mathbf{x}) x_{i}-d_{1}(\mathbf{x}) x_{1}-f_{1}(\mathbf{x}) x_{1} \\
\dot{x}_{2}=f_{1}(\mathbf{x}) x_{1}-d_{2}(\mathbf{x}) x_{2}-f_{2}(\mathbf{x}) x_{2} \\
\vdots \\
\dot{x}_{n}=f_{n-1}(\mathbf{x}) x_{n-1}-d_{n}(\mathbf{x}) x_{n}
\end{array}\right.
$$

This system consists of a single-species population. The individuals in this population are categorized into $n$ classes by developmental stage (or increasing size). $x_{i}$ denotes the population density in stage $i . \beta_{i}(\mathbf{x})$ is the per capita reproduction rate of an individual in stage $i . d_{i}(\mathbf{x})$ is the per capita mortality rate of an individual in stage $i . f_{i}(\mathbf{x})$ is the per capita transition rate from stage $i$ to the next higher stage $i+1$ (note that $f_{n}=0$ ). It is assumed that no individuals can move to the lower stage, and all newborns lie in the first stage.

The matrix $A_{\mathbf{x}}$ of model (5) is given as follows:

$$
A_{\mathbf{x}}=\left(\begin{array}{ccccc}
\beta_{1}(\mathbf{x})-d_{1}(\mathbf{x})-f_{1}(\mathbf{x}) & \beta_{2}(\mathbf{x}) & \cdots & \beta_{n-1}(\mathbf{x}) & \beta_{n}(\mathbf{x}) \\
f_{1}(\mathbf{x}) & -d_{2}(\mathbf{x})-f_{2}(\mathbf{x}) & \cdots & 0 & 0 \\
0 & f_{2}(\mathbf{x}) & \cdots & 0 & 0 \\
\vdots & \vdots & \ddots & \vdots & \vdots \\
0 & 0 & \cdots & f_{n-1}(\mathbf{x}) & -d_{n}(\mathbf{x})
\end{array}\right)
$$

This matrix is divided into two matrices, the fertility matrix $F_{\mathbf{x}}$ and the transition matrix $T_{\mathbf{x}}$ as follows:

$$
F_{\mathbf{x}}=\left(\begin{array}{ccccc}
\beta_{1}(\mathbf{x}) & \beta_{2}(\mathbf{x}) & \cdots & \beta_{n-1}(\mathbf{x}) & \beta_{n}(\mathbf{x}) \\
0 & 0 & \cdots & 0 & 0 \\
0 & 0 & \cdots & 0 & 0 \\
\vdots & \vdots & \ddots & \vdots & \vdots \\
0 & 0 & \cdots & 0 & 0
\end{array}\right)
$$

and

$$
T_{\mathbf{x}}=\left(\begin{array}{ccccc}
-d_{1}(\mathbf{x})-f_{1}(\mathbf{x}) & 0 & \cdots & 0 & 0 \\
f_{1}(\mathbf{x}) & -d_{2}(\mathbf{x})-f_{2}(\mathbf{x}) & \cdots & 0 & 0 \\
0 & f_{2}(\mathbf{x}) & \cdots & 0 & 0 \\
\vdots & \vdots & \ddots & \vdots & \vdots \\
0 & 0 & \cdots & f_{n-1}(\mathbf{x}) & -d_{n}(\mathbf{x})
\end{array}\right)
$$


Note that $T_{\mathbf{x}}=\left(t_{i j}(\mathbf{x})\right)$ contains not only the terms corresponding to transition between stages, but also the terms corresponding to death. So, $\sum_{i=1}^{n} t_{i j}(\mathbf{x})=0$ does not necessarily hold, but $\sum_{i=1}^{n} t_{i j}(\mathbf{x}) \leq 0$ must hold for every $\mathbf{x} \in \mathbb{R}_{+}^{n}$.

We assume the following:

(C1) All functions $\beta_{i}, d_{i}$ and $f_{i}$ are continuously differentiable. All functions $\beta_{i}$ are nonnegative and do not change their sign on $\mathbb{R}_{+}^{n}$. All functions $d_{i}$ and $f_{i}$ are positive.

Then it is clear that the conditions (H1)-(H3) hold. By Proposition 5, we can obtain a sufficient condition for (H4) as follows.

Proposition 14. Assume that $(\mathrm{C} 1)$ holds. If there exist constants $K>0$ and $\lambda_{\infty}<0$ such that $\beta_{i}(\mathbf{x})-d_{i}(\mathbf{x})<\lambda_{\infty}, i=1,2, \ldots, n$, for all $\mathbf{x} \in \mathbb{R}_{+}^{n}$ with $\sum_{i=1}^{n} x_{i} \geq K$, then (5) is dissipative.

REMARK. Theorem 11.2 of [17] gives much more sophisticated results for dissipativity of (5) with $n=2$ and $\beta_{1}=0$.

By applying the results in Sections 4 and 5, we can prove the following proposition.

Proposition 15. Assume that (C1) and (H4) hold.

(i) Suppose that $\beta_{n}$ is positive. If $\mu\left(A_{0}\right)>0$, then (5) is c-permanent.

(ii) Let $J=\{1,2, \ldots, k\}$ for some $1 \leq k \leq n-1$. Suppose that $\beta_{k}$ is positive and $\beta_{k+1}=\beta_{k+2}=\cdots=\beta_{n}=0$. If $\mu\left(A_{0}^{(J)}\right)>0$, then there exists a positive constant $\delta>0$ such that

$$
\liminf _{t \rightarrow \infty} x_{i}(t) \geq \delta, \quad i=1,2, \ldots, n,
$$

for all $\mathbf{x}(0) \in \mathbb{R}_{+}^{n} \backslash O_{J}$.

REMARK. (i) follows from Corollary 9 and Theorem 10, and (ii) follows from Theorems 8 (i), 10 and 11. The schematic portraits of cases (i) and (ii) are depicted in Fig. 1. In case (i), all individuals can reproduce as long as they can attain the final stage. While, in case (ii), individuals cannot reproduce after stage $k$.

The instability of $A_{0}$ (or $A_{0}^{(J)}$ ) can be evaluated by using Theorem 13 . Under the assumption (C1), $F_{0}$ and $T_{0}$ satisfy (A1) and (A2). Note that

$$
-T_{0}^{-1}=\left(\begin{array}{ccccc}
\frac{1}{d_{1}(0)+f_{1}(0)} & 0 & 0 & \cdots & 0 \\
\frac{f_{1}(0)}{\prod_{i=1}^{2}\left(d_{i}(0)+f_{i}(0)\right)} & \frac{1}{d_{2}(0)+f_{2}(0)} & 0 & \cdots & 0 \\
\frac{f_{1}(0) f_{2}(0)}{\prod_{i=1}^{3}\left(d_{i}(0)+f_{i}(0)\right)} & \frac{f_{2}(0)}{\prod_{i=2}^{3}\left(d_{i}(0)+f_{i}(0)\right)} & \frac{1}{d_{3}(0)+f_{3}(0)} & \cdots & 0 \\
\vdots & \vdots & \vdots & \ddots & \vdots \\
\frac{\prod_{i=1}^{n-1} f_{i}(0)}{\prod_{i=1}^{n}\left(d_{i}(0)+f_{i}(0)\right)} & \frac{\prod_{i=2}^{n-1} f_{i}(0)}{\prod_{i=2}^{n}\left(d_{i}(0)+f_{i}(0)\right)} & \frac{\prod_{i=3}^{n-1} f_{i}(0)}{\prod_{i=3}^{n}\left(d_{i}(0)+f_{i}(0)\right)} & \cdots & \frac{1}{d_{n}(0)+f_{n}(0)}
\end{array}\right),
$$


(i)

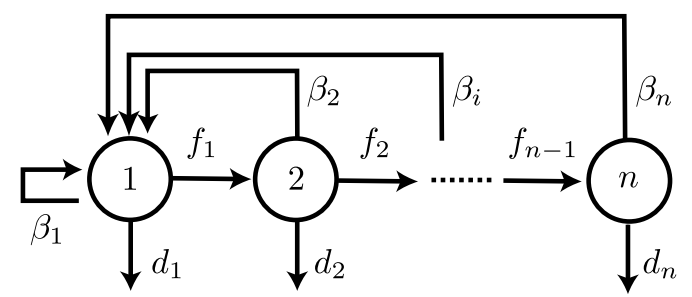

(ii)

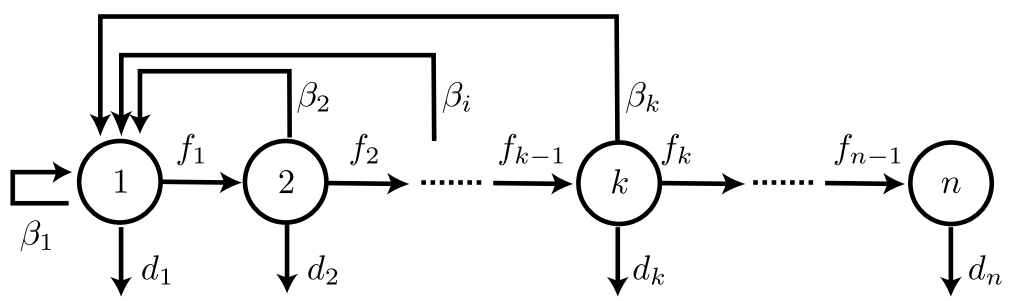

Fig. 1. The schematic portrait of the stage-structured model (5). (i): All individuals can reproduce their offspring as long as they can attain the final stage. (ii): The stages $k+1, k+2, \ldots, n$ are not fertile.

where for notational convenience $f_{n}$ is defined to be 0 . Then $-F_{0} T_{0}^{-1}$ has the eigenvalue

$$
\mathcal{R}_{0}=\sum_{i=1}^{n} \frac{\beta_{i}(0)}{d_{i}(0)+f_{i}(0)} \prod_{j=1}^{i-1} \frac{f_{j}(0)}{d_{j}(0)+f_{j}(0)}
$$

which satisfies $\mathcal{R}_{0}=\mu\left(-F_{0} T_{0}^{-1}\right)$ and whose right eigenvector $\mathbf{w}=(1,0, \ldots, 0)^{\top}$ is nonnegative, i.e., (A3) holds. Moreover, $-T_{0}^{-1} \mathbf{w}>0$. Hence, Theorem 13 ensures that $A_{0}$ is unstable (resp. stable) if $\mathcal{R}_{0}>1$ (resp. $\mathcal{R}_{0}<1$ ). Similarly, the stability of $A_{0}^{(J)}$ is determined by the basic reproduction number of $A_{0}^{(J)}$.

\subsection{Spatially structured models}

Consider the following spatially structured model:

$$
\dot{x}_{i}=g_{i}\left(x_{i}\right) x_{i}+\sum_{j=1}^{n}\left(d_{i j}\left(x_{j}\right) x_{j}-\widetilde{d}_{j i}\left(x_{i}\right) x_{i}\right), \quad i=1,2, \ldots, n,
$$

where $d_{i i}=\widetilde{d}_{i i}=0$. In this model, the population dynamics of a single-species is considered. The habitat for the species is divided into $n$ patches. The population density in patch $i$ is denoted by $x_{i} . g_{i}\left(x_{i}\right)$ is the per capita growth rate in patch $i$ when this patch is isolated from the others. $d_{i j}\left(x_{j}\right)$ and $\widetilde{d}_{i j}\left(x_{j}\right)$ are the immigration and emigration rates from patch $j$ to patch $i$. Since the reproduction during 
dispersal is not considered, $\widetilde{d}_{i j}\left(x_{j}\right) \geq d_{i j}\left(x_{j}\right)$ holds for all $x_{j} \geq 0$. The matrix $A_{\mathbf{x}}$ of this model is

$$
A_{\mathbf{x}}=\left(\begin{array}{cccc}
g_{1}\left(x_{1}\right)-\sum_{j=1}^{n} \widetilde{d}_{j 1}\left(x_{1}\right) & d_{12}\left(x_{2}\right) & \cdots & d_{1 n}\left(x_{n}\right) \\
d_{21}\left(x_{1}\right) & g_{2}\left(x_{2}\right)-\sum_{j=1}^{n} \widetilde{d}_{j 2}\left(x_{2}\right) & \cdots & d_{2 n}\left(x_{n}\right) \\
\vdots & \vdots & \ddots & \vdots \\
d_{n 1}\left(x_{1}\right) & d_{n 2}\left(x_{2}\right) & \cdots & g_{n}\left(x_{n}\right)-\sum_{j=1}^{n} \widetilde{d}_{j n}\left(x_{n}\right)
\end{array}\right) .
$$

We divide this matrix into two matrices as follows:

$$
F_{\mathbf{x}}=\left(\begin{array}{cccc}
g_{1}\left(x_{1}\right) & 0 & \cdots & 0 \\
0 & g_{2}\left(x_{2}\right) & \cdots & 0 \\
\vdots & \vdots & \ddots & \vdots \\
0 & 0 & \cdots & g_{n}\left(x_{n}\right)
\end{array}\right)
$$

and

$$
T_{\mathbf{x}}=\left(\begin{array}{cccc}
-\sum_{j=1}^{n} \widetilde{d}_{j 1}\left(x_{1}\right) & d_{12}\left(x_{2}\right) & \cdots & d_{1 n}\left(x_{n}\right) \\
d_{21}\left(x_{1}\right) & -\sum_{j=1}^{n} \widetilde{d}_{j 2}\left(x_{2}\right) & \cdots & d_{2 n}\left(x_{n}\right) \\
\vdots & \vdots & \ddots & \vdots \\
d_{n 1}\left(x_{1}\right) & d_{n 2}\left(x_{2}\right) & \cdots & -\sum_{j=1}^{n} \widetilde{d}_{j n}\left(x_{n}\right)
\end{array}\right)
$$

We assume the following:

(C2) All functions $g_{i}, d_{i j}$ and $\widetilde{d}_{i j}$ are continuously differentiable. All functions $d_{i j}$ and $\widetilde{d}_{i j}$ are nonnegative, and $\operatorname{sign}\left(d_{i j}\left(x_{j}\right)\right)=\operatorname{sign}\left(d_{i j}(0)\right)=\operatorname{sign}\left(\widetilde{d}_{i j}\left(x_{j}\right)\right)$ and $\sum_{i=1}^{n} \widetilde{d}_{i j}\left(x_{j}\right) \geq \sum_{i=1}^{n} d_{i j}\left(x_{j}\right)$ for all $x_{j} \geq 0$.

Then it is clear that the conditions (H1)-(H3) hold. The following proposition gives a sufficient condition for (H4) (note that we cannot apply Theorem 5 to (6)).

Proposition 16. Assume that (C2) holds. Suppose that there exist positive constants $c, r, K>0$ such that $\max _{i \in N} g_{i}(x) x \leq c$ for all $x \geq 0$ and $\max _{i \in N} g_{i}(x) \leq$ $-r$ for all $x \geq K$. Then (6) is dissipative.

Proof. Let $k=\max \{((n-1) c+\epsilon) / r, K\}$, where $\epsilon>0$ is an arbitrary positive constant. Then, for every $\mathbf{x} \in \mathbb{R}_{+}^{n}$ with $\sum_{i=1}^{n} x_{i} \geq n k$, there exists an $i \in N$ such that $x_{i} \geq k$. Then

$$
\sum_{i=1}^{n} \dot{x}_{i} \leq \sum_{i=1}^{n} g_{i}\left(x_{i}\right) x_{i} \leq(n-1) c-r k \leq-\epsilon
$$




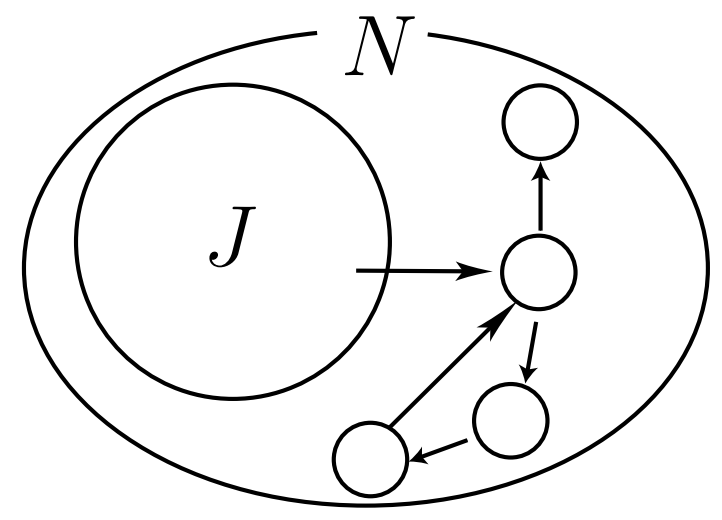

Fig. 2. The schematic portrait of the spatially structured model (6). $J$ is accessible to $N \backslash J$ but $N \backslash J$ is not accessible to $J$ in $G\left(T_{0}\right)$.

holds for all $\mathbf{x} \in \mathbb{R}_{+}$with $\sum_{i=1}^{n} x_{i} \geq n k$. This implies that (6) is dissipative.

By applying the results in Sections 4 and 5, we can obtain the following proposition.

Proposition 17. Assume that (C2) and (H4) hold. Suppose that there exists a nonempty subset $J \subset N$ such that $T_{0}^{(J)}$ is irreducible and $J$ is accessible to $N \backslash J$ but $N \backslash J$ is not accessible to $J$ in $G\left(T_{0}\right)$. If $\mu\left(A_{0}^{(J)}\right)>0$, then there exists a positive constant $\delta>0$ such that

$$
\liminf _{t \rightarrow \infty} x_{i}(t) \geq \delta, \quad i=1,2, \ldots, n
$$

for all $\mathbf{x}(0) \in \mathbb{R}_{+}^{n} \backslash O_{J}$.

REMARK. This proposition follows from Theorems 8 (ii), 10 and 11. The schematic portrait for the situation satisfying the assumptions of the proposition is depicted in Fig. 2. Note that if $T_{0}$ is irreducible, then $\mu\left(A_{0}\right)>0$ implies c-permanence of (6). We can find more sophisticated results for the global dynamics of $(6)$ with constant $d_{i j}$ and $\widetilde{d}_{i j}$ in $[10,13]$.

The instability of $A_{0}^{(J)}$ can be evaluated as follows (in this case Theorem 13 is not useful). Suppose that $T_{0}^{(J)}$ is irreducible. Let $\sum_{i \in J} d_{i j}\left(x_{j}\right)-\sum_{i \in J} \widetilde{d}_{i j}\left(x_{j}\right)=$ $-\epsilon_{j} \leq 0, j \in J$. Then $\sum_{i \in J} a_{i j}(0)=g_{j}(0)-\epsilon_{j}$ for all $j \in J$. Since $A_{0}^{(J)}$ is exponentially nonnegative and irreducible,

$$
\min _{j \in J}\left\{\sum_{i \in J} a_{i j}(0)\right\} \leq \mu\left(A_{0}^{(J)}\right) \leq \max _{j \in J}\left\{\sum_{i \in J} a_{i j}(0)\right\} .
$$

Hence, $\mu\left(A_{0}^{(J)}\right)>0$ if $\min _{j \in J}\left\{g_{j}(0)-\epsilon_{j}\right\}>0$ and $\mu\left(A_{0}^{(J)}\right)<0$ if $\max _{j \in J}\left\{g_{j}(0)-\right.$ 
$\left.\epsilon_{j}\right\}<0$. If $\epsilon_{i}=0$ and $g_{i}(0)=g_{c}$ for all $i \in J$, then $\mu\left(A_{0}^{(J)}\right)>0\left(\right.$ resp. $\left.\mu\left(A_{0}^{(J)}\right)<0\right)$ if and only if $g_{c}>0$ (resp. $\left.g_{c}<0\right)$.

\section{Concluding remarks}

In this paper, we have investigated p- and c-permanence of the structured population model (1). Our results show that, under a certain mild condition, the instability of the population free equilibrium point $\mathbf{x}=0$ implies that the population survives in the sense of c-permanence (note that it is clear that (1) is not c-permanent if $\mathbf{x}=0$ is stable). This result is analogous to the result for the logistic equation, which is an unstructured model.

Our results are applicable even if $A_{0}$ is reducible. By Theorem 8, we can show that an irreducible subsystem of system (1) is c-permanent if the associated submatrix is unstable. Furthermore, by Theorem 11, we can show that every subsystem connecting to the permanent subsystem also survives. However, these theorems are useless to show that all classes in (1) coexist if $G\left(A_{0}\right)$ is not connected (i.e., $G\left(A_{0}\right)$ is split into disconnected groups). In fact, if all individuals in a disconnected subsystem are removed, they cannot reappear by the help of other disconnected subsystems. In this case, we can regard system (1) as a multi-species model. The typical examples are the Lotka-Volterra equations, which have the form (1) with a diagonal matrix $A_{\mathbf{x}}$. It is interesting to extend our results to system (1) with disconnected groups in $G\left(A_{0}\right)$. Furthermore, it is also interesting to consider the extension to a system with inflows from the external system, i.e.,

$$
\dot{\mathbf{x}}=A_{\mathbf{x}} \mathbf{x}+\mathbf{b}(\mathbf{x})
$$

where $\mathbf{b}(\mathbf{x}) \geq 0$ for all $\mathbf{x} \in \mathbb{R}_{+}^{n}$. This type of system often appears as a model of epidemiological or immunological systems (e.g., see $[6,15])$.

\section{Appendix A.}

Consider an ordinary differential equation of the form

$$
\dot{x}_{i}=f_{i}(\mathbf{x}), \quad i=1,2, \ldots, n,
$$

where $\mathbf{x}=\left(x_{1}, x_{2}, \ldots, x_{n}\right)^{\top} \in \mathbb{R}^{n}$ and $f_{i}: \mathbb{R}^{n} \rightarrow \mathbb{R}$. For this equation, we have the following result.

TheOREM 18 (Proposition B.7 of [16]). Suppose that $\mathbf{f}=\left(f_{1}, f_{2}, \ldots, f_{n}\right)^{\top}$ has the property that solutions of initial value problems $\mathbf{x}(0) \in \mathbb{R}_{+}^{n}$ are unique and, for all $i, f_{i}(\mathbf{x}) \geq 0$ whenever $\mathbf{x} \in \mathbb{R}_{+}^{n}$ satisfies $x_{i}=0$. Then $\mathbf{x}(t) \in \mathbb{R}_{+}^{n}$ for all $t \geq 0$ for which it is defined, provided $\mathbf{x}(0) \in \mathbb{R}_{+}^{n}$.

It is well known that if $\mathbf{f}=\left(f_{1}, f_{2}, \ldots, f_{n}\right)^{\top}$ is continuously differentiable and all solutions exist for all time, then the above equation generates a continuous dynamical system $\pi$, i.e., $\pi$ is continuous and satisfies (i) $\pi(\mathbf{x}, 0)=\mathbf{x}$ and (ii) $\pi(\mathbf{x}, t+$ 
$s)=\pi(\pi(\mathbf{x}, t), s)$ for all $s, t \in \mathbb{R}$. The set $\gamma^{+}(\mathbf{x})=\{\mathbf{y}: \pi(\mathbf{x}, t)=\mathbf{y}$ for some $t \geq 0\}$ is called a semi-orbit through $\mathbf{x}$. A set $X \subset \mathbb{R}^{n}$ is said to be forward invariant if $\gamma^{+}(\mathbf{x}) \subset X$ for all $\mathbf{x} \in X$. For subsets $M, Y \subset \mathbb{R}_{+}^{n}, M$ is said to be absorbing for $Y$ if $M$ is forward invariant and $\gamma^{+}(\mathbf{x}) \cap M \neq \emptyset$ for every $\mathbf{x} \in Y$. The $\omega$-limit set of $\mathbf{x}$ is defined by

$$
\omega(\mathbf{x})=\left\{\mathbf{y}: \lim _{j \rightarrow \infty} \pi\left(\mathbf{x}, t_{j}\right) \rightarrow \mathbf{y} \text { for some } t_{j} \rightarrow \infty\right\}
$$

For a subset $X \subset \mathbb{R}^{n}$

$$
\omega(X)=\bigcup_{\mathbf{x} \in X} \omega(\mathbf{x}) .
$$

$S$ is said to be repellor if there exists a neighborhood of $U$ of $S$ such that for all $\mathbf{x} \notin S$ there exists $T=T(\mathbf{x})>0$ satisfying $\pi(\mathbf{x}, t) \notin U$ for all $t \geq T$.

For a continuous dynamical system $\pi$, we have the following theorems.

Theorem 19 (c.f. Lemma 2.1 of [11]). Let $X \subset \mathbb{R}_{+}^{n}$. Let $Y \subset X$ be open, and let $N$ be open with a compact closure $\bar{N} \subset Y$. Assume that $Y$ is forward invariant and that $\gamma^{+}(\mathbf{x}) \cap N \neq \emptyset$ for every $\mathbf{x} \in Y$. Then $M=\gamma^{+}(\bar{N})$ is a compact absorbing set for $Y$.

Theorem 20 (c.f. Theorem 2.5 of [11] and Corollary 2 of [9]). Let $X$ be a compact subset of $\mathbb{R}_{+}^{n}$. Let $S$ be a compact subset of $X$ such that both $X \backslash S$ and $S$ are forward invariant. Then $S$ is a repellor if there exists a continuously differentiable function $P: X \rightarrow \mathbb{R}_{+}$such that (a) $P(\mathbf{x})=0 \Longleftrightarrow \mathbf{x} \in S$ and (b) for all $\mathbf{x} \in \omega(S), \sup _{t \geq 0} \int_{0}^{t} \psi(\mathbf{x}(s)) d s>0$, where $\psi: X \rightarrow \mathbb{R}$ is a continuous function with $\dot{P}(\mathbf{x}) \geq \psi(\mathbf{x}) P(\mathbf{x})$ (" . " denotes differentiation along an orbit).

\section{Appendix B.}

In this Appendix, we list some useful theorems of exponentially nonnegative matrices. It is known that exponentially nonnegative matrices have analogous properties to nonnegative matrices (e.g., see [1, 2, 8, 19] and Appendices in [16, 17] for the theory of nonnegative matrices). We can find some descriptions about exponentially nonnegative matrices in several books (see $[1,19]$ and Appendices in $[16,17]$ ). It is worth noting that the theory of exponentially nonnegative matrices is strongly related to the theory of $M$-matrices (e.g., see Chapter 6 of [2]).

Exponentially nonnegative matrices have the properties stated in the following theorems (Theorem 21 is closely related to the Perron-Frobenius Theorem).

Theorem 21 (c.f. Theorem 8.3 of [19]). Let $A$ be an exponentially nonnegative irreducible $n \times n$ matrix. Then $A$ has a real eigenvalue $\lambda$ such that

(i) To $\lambda$ there corresponds positive right and left eigenvectors.

(ii) $\operatorname{Re} \widetilde{\lambda}<\lambda$ for all other eigenvalues $\tilde{\lambda}$.

(iii) $\lambda$ is algebraically simple. 
REMARK. $\lambda$ is equivalent to $\mu(A), \mu(A)$ is still an eigenvalue of $A$ even if it is reducible. Furthermore, $\mu(A)+s=\rho(A+s I)$ holds for all sufficiently large $s \geq 0$. This can be checked by using the result for nonnegative matrices (e.g., see Theorem 13.3 of [8]).

Theorem 22 (see Chapter 6 of [2]). Let $A$ be an exponentially nonnegative $n \times n$ matrix. $\mu(A)<0$ if and only if $-A^{-1}$ exists and $-A^{-1} \geq 0$.

Remark. See also Lemma of [10] and Theorem A.12 of [16].

\section{Appendix C.}

Theorem 13 is proved by using the following two lemmas.

Lemma 23 (Lemma 6 of [5]). Suppose that $B=\left(b_{i j}\right)$ is a nonnegative $n \times n$ matrix. Then

$$
\rho(B)=\max _{\substack{\mathbf{x} \geq 0 \\ \mathbf{x} \neq 0}} \min _{\substack{i \in N \\ x_{i} \neq 0}} \frac{(B \mathbf{x})_{i}}{x_{i}}=\min _{\substack{\mathbf{x} \geq 0 \\ \mathbf{x} \neq 0}} \max _{i \in N} \frac{(B \mathbf{x})_{i}}{x_{i} \neq 0}
$$

where $(B \mathbf{x})_{i}=\sum_{j=1}^{n} b_{i j} x_{j}$.

Lemma 24. Suppose that $B=\left(b_{i j}\right)$ is an exponentially nonnegative $n \times n$ matrix. Then

$$
\mu(B)=\max _{\substack{\mathbf{x} \geq 0 \\ \mathbf{x} \neq 0}} \min _{\substack{i \in N \\ x_{i} \neq 0}} \frac{(B \mathbf{x})_{i}}{x_{i}}=\min _{\substack{\mathbf{x} \geq 0 \\ \mathbf{x} \neq 0}} \max _{i \in N} \frac{(B \mathbf{x})_{i}}{x_{i} \neq 0}
$$

where $(B \mathbf{x})_{i}=\sum_{j=1}^{n} b_{i j} x_{j}$.

Proof. By the remark of Theorem 21 in Appendix B, $B+s I$ is nonnegative and $\mu(B)+s=\rho(B+s I)$ holds for some $s \geq 0$. Therefore, by Lemma 23, $\rho(B+s I)$ satisfies

$$
\rho(B+s I)=\max _{\substack{\mathbf{x} \geq 0 \\ \mathbf{x} \neq 0}} \min _{\substack{i \in N \\ x_{i} \neq 0}} \frac{((B+s I) \mathbf{x})_{i}}{x_{i}}=\min _{\substack{\mathbf{x} \geq 0 \\ \mathbf{x} \neq 0}} \max _{i \in N} \frac{((B+s I) \mathbf{x})_{i}}{x_{i} \neq 0}
$$

This provides the desired equations.

Proof of Theorem 13. The proof is almost parallel to that in [5].

Note that any eigenvalue of $-F_{0} T_{0}^{-1}$ is also an eigenvalue of $-T_{0}^{-1} F_{0}$ and vice versa, since both matrices have the same characteristic polynomial. Hence, $\mathcal{R}_{0}$ is also an eigenvalue of $-T_{0}^{-1} F_{0}$ and satisfies $\mathcal{R}_{0}=\mu\left(-T_{0}^{-1} F_{0}\right)$. Furthermore, to $\mathcal{R}_{0}$ there corresponds a right eigenvector $-T_{0}^{-1} \mathbf{w}>0$.

By (A1), $\lambda_{0}=\mu\left(A_{0}\right)$ is an eigenvalue of $A_{0}$ and to $\lambda_{0}$ there corresponds a positive right eigenvector $\mathbf{u}>0$. Then $A_{0} \mathbf{u}=\lambda_{0} \mathbf{u}$. Let $G_{0}=-T_{0}^{-1} F_{0}$ and $\widetilde{\mathbf{w}}=-T_{0}^{-1} \mathbf{w}$. By $(\mathrm{A} 3), G_{0} \widetilde{\mathbf{w}}=\mathcal{R}_{0} \widetilde{\mathbf{w}}$ holds. 
Since $A_{0}=F_{0}+T_{0}$ and $G_{0}=-T_{0}^{-1} F_{0}$, the matrices $A_{0}$ and $G_{0}$ satisfy

$$
\begin{aligned}
& A_{0}=-T_{0} G_{0}+T_{0}, \\
& G_{0}=-T_{0}^{-1} A_{0}+I .
\end{aligned}
$$

Therefore, the following equations hold:

$$
\begin{aligned}
& A_{0} \widetilde{\mathbf{w}}=\left(1-\mathcal{R}_{0}\right) T_{0} \widetilde{\mathbf{w}}, \\
& G_{0} \mathbf{u}=-\lambda_{0} T_{0}^{-1} \mathbf{u}+\mathbf{u} .
\end{aligned}
$$

Consequently, for every $i \in N$, we have

$$
\begin{aligned}
& \frac{\left(A_{0} \widetilde{\mathbf{w}}\right)_{i}}{\widetilde{w}_{i}}=\left(1-\mathcal{R}_{0}\right) \frac{\left(T_{0} \widetilde{\mathbf{w}}\right)_{i}}{\widetilde{w}_{i}}, \\
& \frac{\left(G_{0} \mathbf{u}\right)_{i}}{u_{i}}=1+\lambda_{0} \frac{\left(-T_{0}^{-1} \mathbf{u}\right)_{i}}{u_{i}},
\end{aligned}
$$

where $\widetilde{w}_{i}$ and $u_{i}$ are the $i$-th components of $\widetilde{\mathbf{w}}$ and $\mathbf{u}$, respectively. By Lemmas 23 and 24 , we have

$$
\begin{gathered}
\lambda_{0}=\max _{\substack{\mathbf{x} \geq 0 \\
\mathbf{x} \neq 0}} \min _{\substack{i \in N \\
x_{i} \neq 0}} \frac{\left(A_{0} \mathbf{x}\right)_{i}}{x_{i}}=\min _{\substack{\mathbf{x} \geq 0 \\
\mathbf{x} \neq 0}} \max _{\substack{i \in N \\
x_{i} \neq 0}} \frac{\left(A_{0} \mathbf{x}\right)_{i}}{x_{i}}, \\
\mathcal{R}_{0}=\max _{\substack{\mathbf{x} \geq 0 \\
\mathbf{x} \neq 0}} \min _{\substack{i \in N \\
x_{i} \neq 0}} \frac{\left(G_{0} \mathbf{x}\right)_{i}}{x_{i}}=\min _{\substack{\mathbf{x} \geq 0 \\
\mathbf{x} \neq 0}} \max _{\substack{i \in N \\
x_{i} \neq 0}} \frac{\left(G_{0} \mathbf{x}\right)_{i}}{x_{i}} .
\end{gathered}
$$

Note that $G_{0}=-T_{0}^{-1} F_{0}$ is a nonnegative matrix. By using these equations and equations (7) and (8), we obtain the following inequalities:

$$
\begin{gathered}
\min _{i \in N}\left(1-\mathcal{R}_{0}\right) \frac{\left(T_{0} \widetilde{\mathbf{w}}\right)_{i}}{\widetilde{w}_{i}} \leq \lambda_{0} \leq \max _{i \in N}\left(1-\mathcal{R}_{0}\right) \frac{\left(T_{0} \widetilde{\mathbf{w}}\right)_{i}}{\widetilde{w}_{i}}, \\
1+\min _{i \in N} \lambda_{0} \frac{\left(-T_{0}^{-1} \mathbf{u}\right)_{i}}{u_{i}} \leq \mathcal{R}_{0} \leq 1+\max _{i \in N} \lambda_{0} \frac{\left(-T_{0}^{-1} \mathbf{u}\right)_{i}}{u_{i}} .
\end{gathered}
$$

Note that $\max _{i \in N}\left(T_{0} \widetilde{\mathbf{w}}\right)_{i} / \widetilde{w}_{i} \leq 0$ holds since $\left(T_{0} \widetilde{\mathbf{w}}\right)_{i} / \widetilde{w}_{i}=\left(-1 / \mathcal{R}_{0}\right)\left(\left(F_{0} \widetilde{\mathbf{w}}\right)_{i} / \widetilde{w}_{i}\right)$. From (9) and (10), it is clear that $\mathcal{R}_{0}=1$ if and only if $\lambda_{0}=0$. Suppose that $\mathcal{R}_{0}>1$. Then (9) implies $\lambda_{0} \geq 0$ and hence $\lambda_{0}>0$. Conversely, suppose that $\mathcal{R}_{0}<1$. Then (9) implies $\lambda_{0} \leq 0$ and hence $\lambda_{0}<0$. Similarly, by using (10) and $-T_{0}^{-1} \geq 0$, we can show that $\lambda_{0}>0$ (resp. $\left.\lambda_{0}<0\right)$ implies $\mathcal{R}_{0}>1\left(\right.$ resp. $\left.\mathcal{R}_{0}<1\right)$.

The relationship between the basic reproduction number $\mathcal{R}_{0}$ and the instability of the population free equilibrium is applicable to a wide class of structured population models. For example, consider the following SIR epidemiological model:

$$
\left\{\begin{array}{l}
\dot{S}=B-\beta S I-\mu S \\
\dot{I}=\beta S I-\mu I-\alpha I \\
\dot{R}=-\mu R+\alpha I
\end{array}\right.
$$


where $S, I$ and $R$ denotes the population densities of susceptibles, infectives and removed individuals, respectively, and all parameters are positive (e.g., see [6]). The invasibility of the infectious disease is determined by the instability of the disease free equilibrium $(B / \mu, 0,0)$, i.e., the instability of the matrix

$$
A=\left(\begin{array}{cc}
\beta S^{*}-\mu-\alpha & 0 \\
\alpha & -\mu
\end{array}\right) .
$$

Define $F$ and $T$ by

$$
F=\left(\begin{array}{cc}
\beta S^{*} & 0 \\
0 & 0
\end{array}\right), \quad T=\left(\begin{array}{cc}
-\mu-\alpha & 0 \\
\alpha & -\mu
\end{array}\right) .
$$

The positive entry of $F$ denotes the per capita reproduction rate of infectives. The matrix $T$ includes the terms corresponding to death and transition from the class of infectives to the class of removed individuals. The matrices $A, F$ and $T$ satisfy (A1) and (A2), and $-F T^{-1}$ is given as follows:

$$
-F T^{-1}=\left(\begin{array}{cc}
\frac{\beta S^{*}}{\mu+\alpha} & 0 \\
0 & 0
\end{array}\right) .
$$

This has the eigenvalues $\mathcal{R}_{0}=\beta S^{*} /(\mu+\alpha)$ and 0 . We see that $\mathcal{R}_{0}$ is consistent with the biological meaning of the basic reproduction number of infectives. To $\mathcal{R}_{0}$ there corresponds the nonnegative right eigenvector $\mathbf{w}=(1,0)^{\top}$. By Theorem 13, the disease free equilibrium $\left(S^{*}, 0,0\right)$ is unstable (resp. stable) if $\mathcal{R}_{0}>1$ (resp. $\mathcal{R}_{0}<1$ ). Therefore, application of our theorem is not restricted to systems with the form (1).

Acknowledgements. The author would like to thank the anonymous referees for their generous and valuable comments, which greatly improved and simplified the proofs of the theorems. This research is partially supported by the Ministry of Education, Science, Sports and Culture of Japan, Grant-in-Aid for JSPS fellows, 18-9289, 2006.

\section{References}

[1] A. Berman, M. Neumann and R.J. Stern, Nonnegative matrices in dynamic systems. Pure and Applied Mathematics (New York). A Wiley-Interscience Publication, John Wiley \& Sons, Inc., New York, 1989.

[ 2 ] A. Berman and R.J. Plemmons, Nonnegative matrices in the mathematical sciences (Revised reprint of the 1979 original). Classics in Applied Mathematics, 9, Society for Industrial and Applied Mathematics (SIAM), Philadelphia, PA, 1994.

[ 3 ] H. Caswell, Matrix Population Models (second edition). Sinauer Associates, Sunderland, MA, 2001.

[4] J.M. Cushing, An introduction to structured population dynamics. CBMS-NSF Regional Conference Series in Applied Mathematics, 71, Society for Industrial and Applied Mathematics (SIAM), Philadelphia, PA, 1998.

[ 5 ] J.M. Cushing and Z. Yicang, The net reproductive value and stability in matrix population models. Natur. Resource Modeling, 8 (1994), 297-333. 
[6] O. Diekmann and J.A.P. Heesterbeek, Mathematical epidemiology of infectious diseases. Model building, analysis and interpretation. Wiley Series in Mathematical and Computational Biology, John Wiley \& Sons, Ltd., Chichester, 2000.

[7] O. Diekmann, J.A.P. Heesterbeek and J.A.J. Metz, On the definition and the computation of the basic reproduction ratio $R_{0}$ in models for infectious diseases in heterogeneous populations. J. Math. Biol., 28 (1990), 365-382.

[8] F.R. Gantmacher, The theory of matrices, volume 1 (translated from the Russian by K.A. Hirsch, reprint of the 1959 translation). A.M.S. Chelsea Publishing, Providence, RI, 1998.

[ 9 ] J. Hofbauer, A unified approach to persistence. Acta Appl. Math., 14 (1989), 11-22.

[10] J. Hofbauer, An index theorem for dissipative semiflows. Rocky Mountain J. Math., 20 (1990), 1017-1031.

[11] V. Hutson, A theorem on average Liapunov functions. Monatsh. Math., 98 (1984), 267-275.

[12] R. Kon, Y. Saito and Y. Takeuchi, Permanence of single-species stage-structured models. J. Math. Biol., 48 (2004), 515-528.

[13] Z. Lu and Y. Takeuchi, Global asymptotic behavior in single-species discrete diffusion systems. J. Math. Biol., 32 (1993), 67-77.

[14] J.A.J. Metz and O. Diekmann, The dynamics of physiologically structured populations. Lecture Notes in Biomathematics, 68, Springer-Verlag, Berlin, 1986.

[15] M.A. Nowak and R.M. May, Virus dynamics: mathematical principles of immunology and virology. Oxford University Press, Oxford, 2000.

[16] H.L. Smith and P. Waltman, The theory of the chemostat, Dynamics of microbial competition, Cambridge Studies in Mathematical Biology, 13, Cambridge University Press, Cambridge, 1995.

[17] H.R. Thieme, Mathematics in population biology. Princeton Series in Theoretical and Computational Biology, Princeton University Press, Princeton, NJ, 2003.

[18] P. van den Driessche and J. Watmough, Reproduction numbers and sub-threshold endemic equilibria for compartmental models of disease transmission. Math. Biosci., 180 (2002), 29-48.

[19] R.S. Varga, Matrix iterative analysis (second revised and expanded edition). Springer Series in Computational Mathematics, 27, Springer-Verlag, Berlin, 2000 
